\title{
SELEÇÃO DE FUNGOS FILAMENTOSOS PARA PRODUÇÃO DE HIDROLASES LIGNOCELULOLÍTICAS POR FERMENTAÇÃO SUBMERSA COM USO DE RESÍDUOS DA AGROINDÚSTRIA DE ÓLEOS VEGETAIS COMO FONTE DE CARBONO
}

\author{
R. S. SANTOS ${ }^{1,2}$, F.K.P. ALVES ${ }^{3}$, A. P. F. C. VANZELA ${ }^{1,2}$, L. A. PANTOJA ${ }^{2,4}$, A. S. SANTOS ${ }^{2,3}$ \\ ${ }^{1}$ Departamento de Farmácia - Universidade Federal dos Vales do Jequitinhonha e Mucuri - UFVJM \\ ${ }^{2}$ Programa de Pós-graduação em Biocombustíveis - UFVJM, \\ ${ }^{3}$ Departamento de Ciências Básicas - UFVJM \\ ${ }^{4}$ Instituto de Ciências e Tecnologia - UFVJM
}

E-mail para contato: ricardo.santos@ufvjm.edu.br

\begin{abstract}
RESUMO - Pesquisas voltadas para produção de enzimas lignocelulolíticas constituem-se em valorosa ação para o desenvolvimento no setor de biocombustíveis, em especial para a produção de etanol de $2^{a}$ Geração. Neste contexto, o presente trabalho teve por objetivo avaliar a produção de celulases e xilanases por fungos filamentosos em fermentação submersa, utilizando resíduos da cadeia produtiva do biodiesel como fontes de carbono. Três linhagens isoladas de amostras ambientais e uma linhagem de referência, Trichoderma reesei CCT2768, foram avaliadas para produção das enzimas supracitadas. Dentre os micro-organismos avaliados, um isolado identificado como Aspergillus tubingensis AN1257 se destacou para a produção das enzimas investigadas, utilizando torta de caroço de algodão, exibindo valores de $694 \mathrm{U} / \mathrm{L}$ de atividade de endoglucanase, $620 \mathrm{U} / \mathrm{L}$ de $\beta$-glucosidase e 48 U/L de FPase após o período de 96 horas de fermentação, e $30 \mathrm{U} / \mathrm{mL}$ de atividade xilanolítica após 72 horas de fermentação.
\end{abstract}

\section{INTRODUÇÃO}

Enzimas celulolíticas e xilanolíticas têm sido comumente utilizadas nas indústrias têxteis, de papel e celulose, de alimentos e bebidas, e de ração animal. Entretanto, nos últimos anos houve maior interesse por estudos e pesquisas sobre estas enzimas visando o aproveitamento de biomassas lignocelulósicas. Este fato deve-se principalmente aos avanços industriais que geram diariamente toneladas de resíduos sólidos lignocelulósicos, os quais muitas vezes, se tornam um problema ecológico (Sukumarana, et al., 2009. Visser et al., 2011).

A demanda por estes catalisadores lignocelulolíticos vem crescendo rapidamente e impulsionando pesquisas relacionadas ao uso de celulases e xilanases na produção de etanol de $2^{\mathrm{a}}$ Geração (Camassola e Dillon, 2009; Gottschalk et al., 2010; Maeda et al., 2013). Contudo, o atual 


\section{9 a 22 de outubro de 2014 \\ Florianópolis/SC}

emprego de enzimas comerciais neste processo, denominado sacarificação enzimática, apresenta alto custo, o que torna a produção do etanol de segunda geração mais onerosa (Fachinni et al., 2011). A fim de contornar este problema, um dos focos para o desenvolvimento tecnológico de obtenção do etanol lignocelulósico está na produção de enzimas de baixo custo. Parte deste caminho passa pela seleção de micro-organismos produtores de enzimas lignocelulolíticas, dentre os quais, os fungos filamentosos são especialmente importantes. Outro fato relevante para a produção de enzimas é a composição dos meios de fermentação, que contribui consideravelmente para o preço final do produto tornando necessária a busca por substratos de baixo custo (Fachinni et al., 2011).

Neste contexto, o presente trabalho teve por objetivo avaliar o potencial indutor das tortas de algodão, girassol e macaúba, resíduos da cadeia de produção do biodiesel, para produção de celulases e xilanases por novos isolados de fungos filamentosos quando utilizadas como fontes de carbono em processo de fermentação submersa.

\section{METODOLOGIA}

\subsection{Obtenção e preparo das tortas}

A torta de algodão utilizada neste estudo foi doada pela Indústria de Óleo, Rações e Plásticos Montes Claros LTDA - localizada no município de Montes Claros, MG; a torta de girassol foi doada pela empresa BIOSEP, localizada no município de Três Pontas, MG; e a torta de macaúba foi doada pela Unidade de Beneficiamento do Coco de Macaúba - UBCM, localizada na cidade de Mirabela MG.

\subsection{Obtenção e manutenção das linhagens de fungos}

As três linhagens de fungos filamentosos utilizadas neste estudo Aspergillus tubingensis AN1257, Penicillium sp T1.1 e PV (linhagem não identificada) foram escolhidas a partir de resultados de triagem realizada anteriormente. Para fins comparativos foi utilizada uma linhagem de Trichoderma reesei CCT2768, reconhecida como excelente produtora de celulases. As linhagens foram mantidas no Laboratório de Bioprocessos e Biotransformação - UFVJM, estocadas em tubos contendo meio complexo de extrato de aveia para a linhagem Trichoderma reesei e meio PDA (potato dextrose ágar) para as demais culturas. As linhagens estudadas foram cultivadas a $30^{\circ} \mathrm{C}$ durante 14 dias para linhagem de Trichoderma reesei e 7 dias para as demais. Para manutenção, foram realizados repiques periódicos (a cada 3 meses) sendo as culturas conservadas sob refrigeração $\left(4 \pm 1^{\circ} \mathrm{C}\right)$.

\subsection{Processo fermentativo para produção de enzimas lignocelulolíticas}

As culturas estoque foram repicadas em placas de Petri contendo meio Vogel (Vogel, 1964), em triplicata, e incubadas a $30^{\circ} \mathrm{C}$ por 14 (Trichoderma reesei) e 7 dias (PV, T1.1, AN1257). Os conídios foram coletados assepticamente em $10 \mathrm{~mL}$ de água destilada estéril, filtrados através de gaze e contados em Câmara de Neubauer para determinação da concentração do inóculo.

Os fungos filamentosos estudados foram avaliados quanto ao desempenho no processo de 


\section{9 a 22 de outubro de 2014 \\ Florianópolis/SC}

fermentação submersa (FSb) em meios elaborados com torta de algodão, torta de girassol, torta de macaúba e celulose microcristalina comercial como fontes de carbono.

O experimento foi realizado de modo a avaliar a produção de endoglucanase e xilanase. O processo fermentativo foi conduzido por 120 horas, em frascos cônicos de $125 \mathrm{~mL}$, em triplicata, contendo $25 \mathrm{~mL}$ de meio base composto de extrato de levedura $(0,4 \mathrm{~g} / \mathrm{L}), \mathrm{NaCl}(0,1 \mathrm{~g} / \mathrm{L}), \mathrm{MgSO}_{4^{-}}$ $7 \mathrm{H}_{2} \mathrm{O}(0,2 \mathrm{~g} / \mathrm{L}), \mathrm{KH}_{2} \mathrm{PO}_{4}(0,4 \mathrm{~g} / \mathrm{L}), \mathrm{K}_{2} \mathrm{HPO}_{4}(0,1 \mathrm{~g} / \mathrm{L})$ e $5 \mathrm{~g}$ de uma das fontes de carbono supracitadas. Em cada processo, separadamente, foram utilizados como agentes de fermentação as linhagens de fungos filamentosos AN1257, T1.1, PV e a linhagem controle. Cada suspensão de conídios foi inoculada de forma a obter uma concentração final de $1,0 \times 10^{5}$ conídios $/ \mathrm{mL}$ de meio de fermentação (meio base). O processo fermentativo foi conduzido a $30^{\circ} \mathrm{C}$ em estufa incubadora, marca Nova Ética ${ }^{\circ}$, sob agitação a 150 rpm.

A obtenção dos extratos enzimáticos foi realizada por meio de filtração em funil de Büchner à vácuo utilizando papel de filtro, previamente pesado, com retenção de partículas de 7 a $12 \mu \mathrm{m}$. $\mathrm{O}$ filtrado foi recolhido em tubos de ensaio imersos em gelo em escama contido dentro de Kitassato. $\mathrm{O}$ filtrado foi mantido em gelo até a determinação da atividade das enzimas estudadas. $\mathrm{O}$ papel de filtro contendo o resíduo sólido do meio fermentativo foi seco em estufa a $60^{\circ} \mathrm{C}$, pesado e os valores utilizados para a determinação de peso seco.

Após avaliação das linhagens e fontes de carbono estudadas, uma nova fermentação foi conduzida, utilizando apenas a linhagem e fonte de carbono que se destacaram, na qual foram determinadas as atividades de endoglucanase, $\beta$-glucosidase, FPase, xilanase (Ghose, 1987) e protease. Foi ainda, quantificado o teor de proteínas solúveis (Lowry, 1951), açúcares redutores (Miller, 1959), peso seco e determinado o pH do meio a cada 24 horas, em um período de 144 horas de fermentação submersa.

\section{RESULTADOS E DISCUSSÕES}

\subsection{Processo fermentativo para produção de enzimas lignocelulolíticas}

O gráfico representado pela Figura 1 ilustra as atividades máximas de endoglucanase (U/L) dos extratos brutos produzidos pelas linhagens AN1257 (Aspergillus tubingensis), T1.1 (Penicillium sp.), PV (linhagem não identificada) e Trichoderma reesei CCT2768, por processo de fermentação submersa (FSb) em meios elaborados com torta de algodão, torta de girassol, torta de macaúba e celulose microcristalina comercial como fontes de carbono. Observa-se que dentre as três linhagens avaliadas, a que melhor produziu a enzima endoglucanase foi a linhagem Aspergillus tubingensis AN1257, com atividade de 713 U/L, após 96 horas de fermentação, valor próximo ao encontrado por Shimokawa (2012) que avaliou esta mesma espécie. Pode-se observar ainda, que a produção de endoglucanase pela linhagem AN1257 foi superior em 13,6 \% em relação à linhagem de referência, Trichoderma reesei CCT2768, que apresentou uma atividade máxima de 616U/L de endoglucanase após 96 horas de fermentação. 


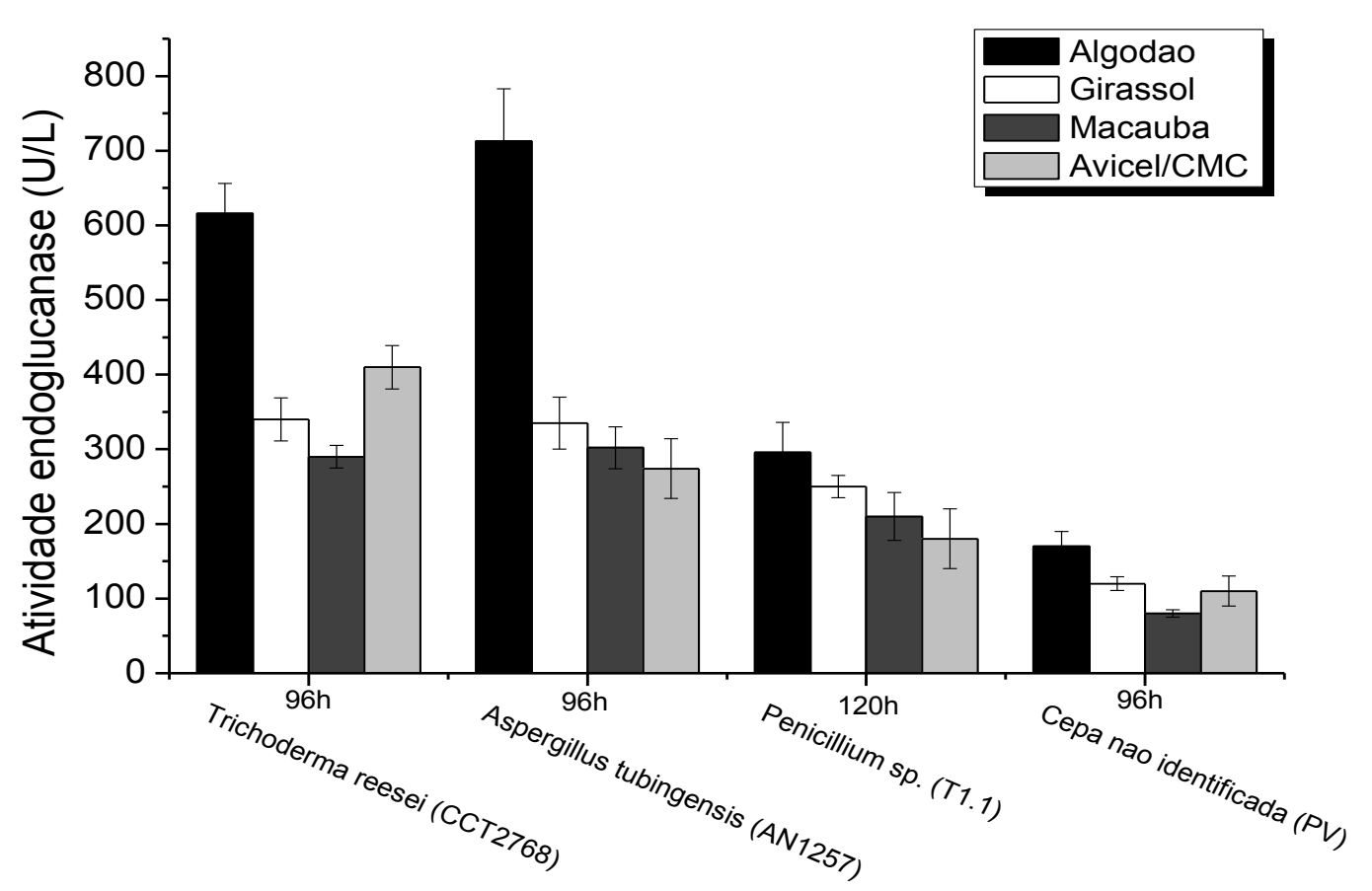

Figura 1 - Valores de produção máxima da enzima endoglucanase pelas linhagens Trichoderma reesei CCT2768, Aspergillus tubingensis AN1257, Penicillium sp. T1.1, e PV, em processo de fermentação submersa

Quanto às fontes de carbono utilizadas, a torta de algodão foi a que melhor induziu a produção de endoglucanase, tanto pela linhagem de Aspergillus tubingensis AN1257 quanto pela linhagem de Trichoderma reesei CCT2768. Alguns resíduos das indústrias de biodiesel têm sido relatados como fontes de carbono para produção de enzimas lignocelulolíticas, como torta de dendê (Alam et al., 2005), resíduos de amendoim (Vyas e Vyas, 2005), torta de pinhão-manso (Ncube et al., 2012), torta de mamona (Herculano et al., 2011). A utilização de resíduos da cadeia do biodiesel em bioprocessos para obtenção de enzimas tem se mostrado como ferramenta economicamente promissora, pois além agregar valores aos coprodutos, permitirá a redução do custo do processo de produção de enzimas hidrolíticas.

Dentre as linhagens avaliadas, o Aspergillus tubingensis AN1257 também se mostrou um excelente produtor de xilanases, produzindo nesta etapa do estudo $30 \mathrm{U} / \mathrm{mL}$ de atividade xilanolítica. Porém, a linhagem de referência (Trichoderma reesei CCT2768) produziu maior atividade, chegando a atingir 39U/mL de atividade xilanolítica nas mesmas condições fermentativas aplicadas para o Aspergillus tubingensis AN1257 (Figura 2). A torta de algodão também foi a melhor opção como fonte de carbono para produção de enzimas xilanolíticas. Vale ressaltar que esta biomassa induziu melhor a produção de enzimas xilanolíticas, mesmo apresentando quantidades inferiores de hemicelulose $(15,19 \pm 1,49 \%)$ quando comparada à torta de girassol $(20,55 \pm 1,22 \%)$. Desse modo, o fungo filamentoso Aspergillus tubingensis AN1257 e a torta de algodão foram escolhidos para dar continuidade ao estudo. 


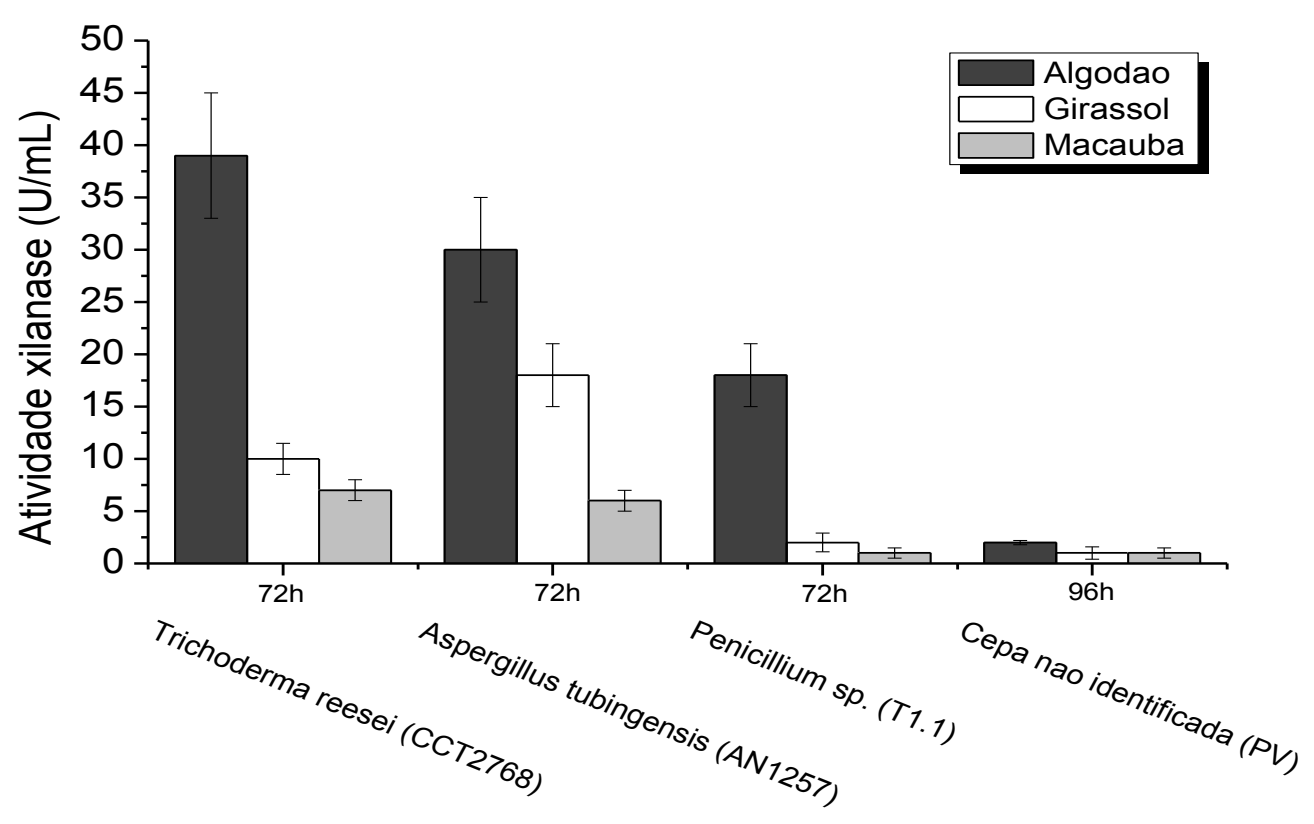

Figura 2 - Valores de produção máxima da enzima xilanase pelas linhagens Trichoderma reesei CCT2768, Aspergillus tubingensis AN1257, Penicillium sp. T1.1 e PV (linhagem não identificada) em processo de fermentação submersa

Os gráficos representados na Figura 3 demonstram o perfil fermentativo para produção de enzimas lignocelulolíticas, no qual estão ilustrados as atividades de endoglucanase, $\beta$-glucosidase, fpase, xilanase, além das atividades de protease, produção de proteínas solúveis, consumo de açúcares redutores, variação de $\mathrm{pH}$ e peso seco, no período de 144 horas de fermentação submersa, utilizando torta de algodão como fonte de carbono e a linhagem de Aspergillus tubingensis AN1257. Foi possível observar que a linhagem em estudo apresentou considerável capacidade de produção para as enzimas celulolíticas e xilanolíticas, exibindo valores de $694 \mathrm{U} / \mathrm{L}$ de atividade de endoglucanase, 620 U/L de $\beta$-glucosidase e $48 \mathrm{U} / \mathrm{L}$ de FPase após o período de 96 horas de fermentação e $30 \mathrm{U} / \mathrm{mL}$ de atividade xilanolítica após 72 horas de fermentação (Figura 3). O tempo de 96 horas para produção máxima de enzimas celulolíticas por linhagens do gênero Aspergillus sp. também foi observado por Kang et al., 2004 e Bansal et al., 2012. A atividade máxima de xilanase foi observada em um período menor que o observado para a produção de celulases, resultados condizentes aos relatados por Facchini 2011. Nestes mesmos gráficos (Figura 3) também foi apresentado o perfil de produção de proteína solúvel $(\mathrm{g} / \mathrm{L})$. O aumento de proteína solúvel no extrato enzimático ocorreu antes do aparecimento das atividades celulásicas (Figura 3), indicando que a detecção do aumento de proteínas pode estar associada principalmente à produção de enzimas xilanolíticas (Figura 3). Com base nos valores de proteína solúvel pode-se calcular os valores de atividade enzimática específica (U/g de proteína solúvel) obtendo atividades de aproximadamente $875 \mathrm{U} / \mathrm{g}$ de proteína solúvel para endoglucanase, $785 \mathrm{U} / \mathrm{g}$ de proteína solúvel para $\beta$-glucosidase, $60 \mathrm{U} / \mathrm{g}$ de proteína solúvel para Fpase e $70 \mathrm{U} / \mathrm{mg}$ de proteína solúvel para xilanase. No perfil de produção de proteases, representado na Figura 3, é possível observar a presença de enzimas proteolíticas com atividade de 100 U/L após 120 
horas de processo fermentativo, fato que possivelmente contribuiu para a redução das atividades das enzimas xilanolíticas e celulolíticas como apresentado na Figura 3.

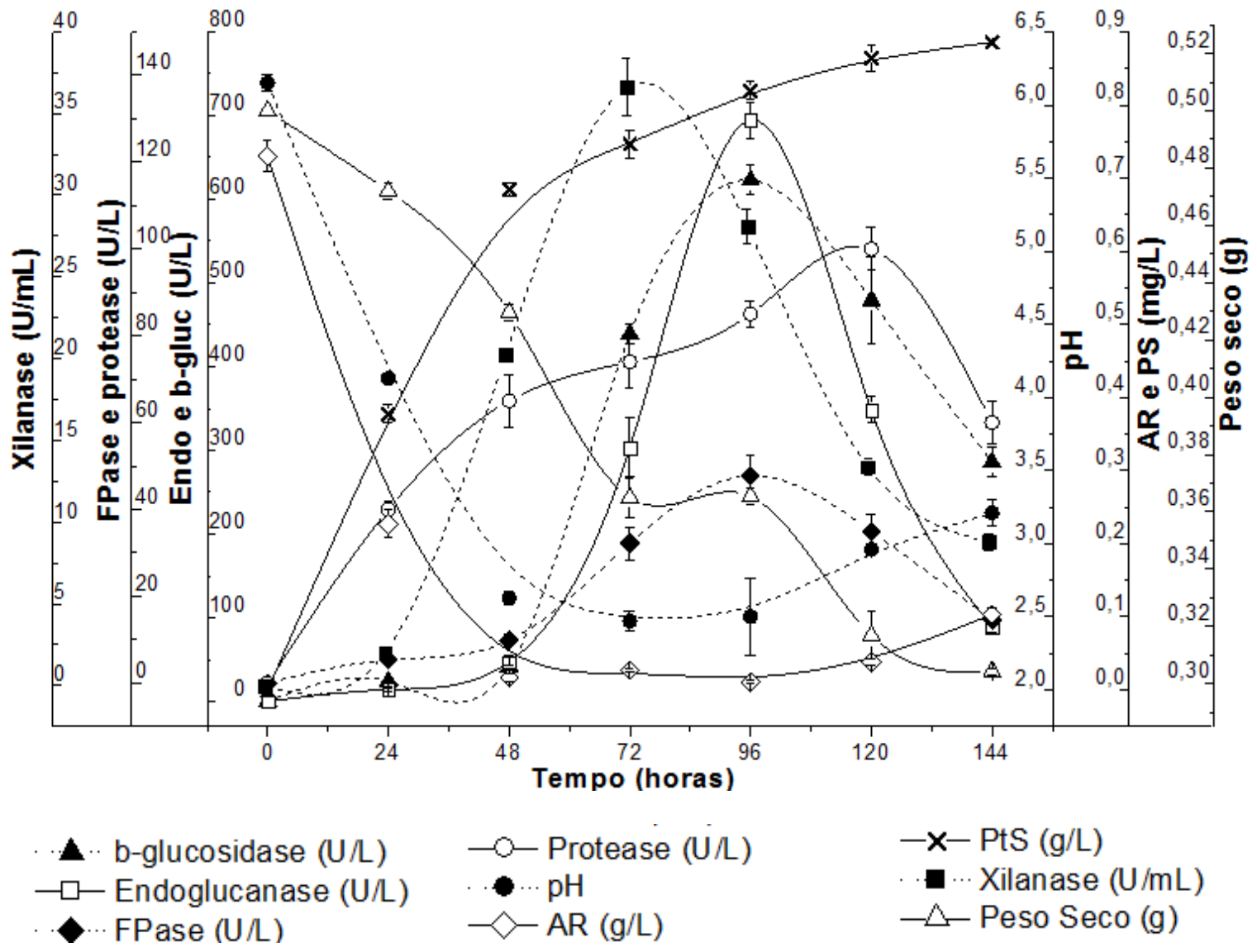

Figura 3 - Perfis de atividade das enzimas endoglucanase, $\beta$-glucosidase, FPase, Xilanase, protease, proteínas solúveis (PtS), consumo de açúcares redutores (AR), variação de pH e peso seco, no período de 144 horas de fermentação submersa, utilizando torta de algodão e a linhagem Aspergillus tubingensis AN1257.

Neste experimento também foi possível avaliar alguns parâmetros bioquímicos e fisiológicos como a alteração de $\mathrm{pH}$ do meio durante o processo fermentativo, consumo de açúcares redutores disponíveis no meio e variação de peso seco. Durante o processo de fermentação submersa com a linhagem de Aspergillus tubingensis AN1257 houve uma redução significativa do pH do meio (Figura 3). Os dados evidenciam que no pico de produção enzimática, 72 horas para xilanase e 96 horas para celulases, que podem ser observados na Figuras 3, o valor de $\mathrm{pH}$ decaiu bruscamente, ficando em torno de 2,5. Tais dados são concordantes com o fato de que várias espécies de Aspergillus negros, como o A. tubingensis da linhagem AN1257, são produtores de ácidos orgânicos e, geralmente, precisam de um $\mathrm{pH}$ fortemente ácido para o seu crescimento. Diante destes resultados, surge a 
necessidade de estudos sobre a influência do $\mathrm{pH}$ inicial do meio de cultivo para produção de celulases e xilanases por Aspergillus tubingensis AN1257. O perfil de utilização dos açúcares redutores solúveis no meio fermentativo pela linhagem em estudo (Figura 3) permite sugerir que a disponibilidade inicial de açúcares, prontamente assimiláveis, quando comparado com a curva de produção das enzimas do complexo das celulases, promove uma repressão na produção destas enzimas, a qual é iniciada imediatamente após o consumo dos mesmos.

Observou-se que os resultados deste estudo foram superiores a diversos estudos relatados nos últimos anos (Tabela 1) relacionados à produção de endoglucanase e xilanase por linhagens de fungos do gênero Aspergillus em processo de fermentação submersa, empregando resíduos lignocelulósicos como fonte de carbono.

Tabela 1 - Informações sobre estudos científicos realizados sobre a produção de enzimas celulolíticas e xilanolíticas por fungos do gênero Aspergillus spp. em fermentação submersa, utilizando diferentes resíduos lignocelulósicos como fonte de carbono.

\begin{tabular}{|c|c|c|c|c|}
\hline & Fonte Carbono & $\begin{array}{c}\text { Endoglucanase } \\
(\mathrm{U} / \mathrm{L})\end{array}$ & $\begin{array}{l}\text { Xilanase } \\
\text { (U/mL) }\end{array}$ & Referência \\
\hline A. niger & $\begin{array}{c}\text { Bagaço de cana de } \\
\text { açúcar }\end{array}$ & - & 1,96 & Cunha et al. 2012 \\
\hline A. japonicus & $\begin{array}{l}\text { Sabugo de milho + } \\
\text { farelo de soja }\end{array}$ & - & 26,2 & Facchini et al. 2011 \\
\hline A. terreus & Farelo Mamona & 630 & - & Herculano et al. 2011 \\
\hline A. candidus & Farelo Mamona & 530 & - & Herculano et al. 2011 \\
\hline A. japonicus & Farelo Mamona & 0,37 & - & Herculano et al. 2011 \\
\hline
\end{tabular}

\section{CONCLUSÕES}

Apesar de celulases e xilanases serem comumente comercializadas, vale ressaltar que a produção brasileira destas enzimas não atende a demanda nacional havendo necessidade de importação das mesmas. Diante do exposto, há necessidade de estudos voltados para a produção de enzimas lignocelulolíticas a fim de torná-las mais acessíveis e com baixo custo. A utilização de resíduos provenientes da extração lipídica de oleaginosas, oriundos da cadeia de produção do biodiesel, como fonte de carbono em processos de fermentação por fungos filamentosos se mostrou relevante para produção de celulases e xilanases,. O estudo revelou novas linhagens de microorganismos com potencial biotecnológico. Por fim, a pesquisa realizada gerou perspectivas de novos estudos relacionados ao aproveitamento de resíduos da cadeia do biodiesel para a produção de bioetanol e hidrolases lignocelulolíticas por processos de baixo custo.

\section{REFERÊNCIAS}

ALAM, M.Z.; MUHAMMAD, N.; MAHAMAT, M.E. Production of cellulase from oil palm biomass as substrate by solid state bioconversion. Am. J. Appl. Sci. v. 2, p. 569-572, 2005.

BANSAL, N.; TEWARI, R.; SONI R; SONI, S.K. Production of cellulases from Aspergillus niger 


\section{9 a 22 de outubro de 2014 \\ Florianópolis/SC}

NS-2 in solid state fermentation on agricultural and kitchen waste residues. Waste Management, v. 32, p. 1341-1346, 2012.

CAMASSOLA, M. DILLON, A.J.P. Biological pretreatment of sugar cane bagasse for the production of cellulases and xylanases by Penicillium echinulatum. Ind. crops and products. v. 29, p. 642-647, 2011.

CUNHA, F. M., et al. Sequential solid-state and submerged cultivation of Aspergillus niger on sugarcane bagasse for the production of cellulase. Bioresour. Technol. 2012.

FACCHINI, F. D. A.; VICI, A. C.; BENASSI, V. M.; FREITAS, L. A. P.; REIS, R. A.; JORGE, J. A.; TERENZI, H. F.; POLIZELI M. L. T. M. Optimization of fibrolytic enzyme production by Aspergillus japonicus $\mathrm{C} 03$ with potential application in ruminant feed and their effects on tropical forages hydrolysis. Bioprocess Biosyst Eng, 2011.

GHOSE, T. K. Measurement of cellulose activities. Pure and Ap. Chem., v. 59, p. 257-268, 1987.

GOTTSCHALK, L.M.F. OLIVEIRA, R.A., BOM, E. P. S. Cellulases, xylanases, -glucosidase and ferulic acid esterase produced by Trichoderma and Aspergillus act synergistically in the hydrolysis of sugarcane bagasse. Biochemical Engineering Journal. 51, 72-78, 2010.

HERCULANO, P. N. et al. Cellulolytic Fungi from Castor Curr Microbiol v. 62, p. 1416-1422, 2011. LOWRY, O.H.; ROSEBROUGH, N.J.; FARR, A.L.; RANDALL, R.J. Protein measurement with the folin phenol reagent.JBiolChem, v. 193, p. 265- 275, 1951.

MAEDA, R.N.; BARCELOS, C.A.; SANTAANNA L.M.M.; PEREIRA JR, N. Cellulase production by Penicillium funiculosum and its application in the hydrolysis of sugar cane bagasse for second generation ethanol production by fed batch operation. J. of Biotechnology, v. 163, p. 38-44, 2013.

MILLER, G. L. Use of dinitrosalicylic acid reagent for determination of reducing sugar. Anal. Chem., v.31, p.426-428, 1959.

NCUBE T., RACHMOND L. H., ABOTSI E. K., RENSBURG E. L. J., NCUBE I. Jatropha curcas seed cake as substrate for production of xylanase and cellulase by Aspergillus niger FGSCA733 in solid-state fermentation. Industrial Crops and Products, v. 37, p. 118- 123, 2012.

KANG, S.W.; PARK, Y.S.; LEE, J.S.; HONG, S.I.; KIM, S.W. Production of cellulases and hemicellulases by Aspergillus niger KK2 from lignocellulosic biomass. Bioresource Technology. v. 91, p. 153-156, 2004.

SUKUMARAN, R. K.; SINGHANIA, R. R.; MATHEW, G. M.; PANDEY, A. Cellulase production using biomass feed stock and its application in lignocellulose saccharification for bio-ethanol production. Renewable Energy, v.34, p.421-424, 2009.

VISSER E.M., OLIVEIRA FILHO, D., MARTINS,M.A., BRIAN L. STEWARD. Bioethanol production potential from Brazilian biodiesel co-products. Biomass and Bioenergy 35,489-494, 2011. VOGEL, H. F. Distribution of lysine pathways among fungi: evolutionary implications. AmerNaturalist, v. 98, p. 435-446, 1964.

VYAS, A., VYAS, D.,. Production of fungal cellulases by solid state bioprocessing of groundnut shell wastes. J. Sci. Ind. Res. 64, 767-770, 2005. 